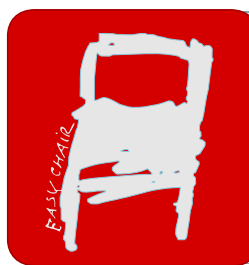

EPiC Series in Health Sciences

Volume 4, 2020, Pages 126-130

CAOS 2020. The 20th Annual Meeting of the International Society for Computer Assisted Orthopaedic Surgery

\title{
Effect of GAN-based image standardization on MR knee bone-tissue classification performance
}

\author{
Vincent Jaouen ${ }^{1 *}$ Guillaume Dardenne ${ }^{1}$, Florent Tixier $^{1}$, Éric Stindel ${ }^{1}$, and \\ Dimitris Visvikis ${ }^{1}$ \\ UMR U1101 LaTIM, Inserm, Université de Bretagne Occidentale, IMT Atlantique, CHRU Brest \\ Brest, France \\ vjaouen@gmail.com
}

\section{Introduction}

Due to their sensitivity to acquisition parameters, medical images such as magnetic resonance images (MRI), Positron Emission tomography (PET) or Computed Tomography (CT) images often suffer from a kind of variability unrelated to diagnostic power, known as the center effect (CE) or domain shift. This is especially true in MRI, where units are arbitrary and image values can strongly depend on subtle variations in the pulse sequences [2]. Due to the CE it is particularly difficult in various medical imaging applications to 1) pool data coming from several centers or 2) train machine learning algorithms requiring large homogeneous training sets. There is therefore a clear need for image standardization techniques aiming at reducing this effect. Considerable improvements in image synthesis have been achieved over the recent years using (deep) machine learning. Models based on generative adversarial neural networks (GANs) now enable the generation of high definition images capable of fooling the human eye [5]. These methods are being increasingly used in medical imaging for various cross-modality (image-to-image) applications such as MR to CT synthesis [3]. However, they have been seldom used for the purpose of image standardization, i.e. for reducing the CE [4]. In this work, we explore the potential advantage of embedding a standardization step using GANs prior to knee bone tissue classification in MRI. We consider image standardization as a within-domain image synthesis problem, where our objective is to learn a mapping between a domain $\mathcal{D}$ constituted of heterogeneous images and a reference domain $\mathcal{R}$ showing one or several images of desired image characteristics.

\section{Methods}

Eleven T1-weighted knee MR images were collected from the Brest University Hospital, France (denoted as $\mathcal{I}_{01}$ to $\mathcal{I}_{11}$ ). Images belonged to different studies and were produced using different pulse sequences, showing a large variability in terms of properties such as slice thickness,

* Corresponding author

F. Rodriguez Y Baena and F. Tatti (eds.), CAOS 2020 (EPiC Series in Health Sciences, vol. 4), pp. 126-130 
in-plane resolution or bone-to-soft tissue contrast (Fig. 1a). To perform image standardization, we trained a 2D CycleGAN neural network architecture [7] on sagittal slices to learn a mapping between $\mathcal{I}_{05}$, taken arbitrarily as the reference domain $\mathcal{R}$, and the remaining subjects constituting domain $\mathcal{D}$. Due to the unbalanced nature of the two domains, in order to reduce overfitting of $\mathcal{D}$ to $\mathcal{R}$, data augmentation of $\mathcal{R}$ was performed using 3D rotations and flipping prior to $2 \mathrm{D}$ slicing. To further prevent overfitting, an unusually short training time of 30 epochs was set using Adam optimizer, based on our prior observations that image style is transferred before structure [4]. The trained network weights were then applied in a feed forward fashion for inference on the remaining images. Note that no distinction was made between training and testing set due to the nature of the objective pursued.

\section{Experiments and results}

To evaluate the effect of image standardization on bone tissue classification, we studied the performance of a random-forest classifier [1] trained using bone intensity values of $\mathcal{I}_{05}$ on the remaining subjects before and after the proposed standardization step. For evaluation purposes, bones were manually segmented by an expert using ITK-SNAP [6] to produce segmentation masks for all eleven subjects. After standardization, image quality was visually more satisfying with improved homogeneity in image properties such as similar bone-to-soft tissue contrast across subjects (Fig. 1b). No noticeable overfitting to $\mathcal{I}_{05}$ could be observed suggesting good preservation of individual structures. The bone probability maps showed a stronger agreement with actual bone tissue (Fig. 2a) compared to non-standardized data, for which the bone tissue was almost completely missed in some subjects (Fig. 2a). These observations were confirmed quantitatively with an average bone probability of $0.16 \pm 0.18$ without standardization against $0.43 \pm 0.04$ after the proposed standardization step.

\section{Conclusion}

We have studied the impact of a novel image standardization technique based on deep generative learning to reduce the center effect in knee MR images for improved automatic bone-tissue classification. Preliminary results using random forest classification showed a clear beneficial impact of the proposed GAN-based image standardization approach. Further experiments will be conducted to validate the method more extensively using fully automatic bone segmentation pipelines based on recent deep learning segmentation architectures.

\section{Acknowledgments}

This work benefited from state aid managed by the National Research Agency under the future investment program bearing the reference ANR-17-RHUS-0005.

\section{References}

[1] Antonio Criminisi, Jamie Shotton, and Ender Konukoglu. Decision forests: A unified framework for classification, regression, density estimation, manifold learning and semi-supervised learning. Foundations and Trends(R) in Computer Graphics and Vision, 7(2-3):81-227, 2012. 


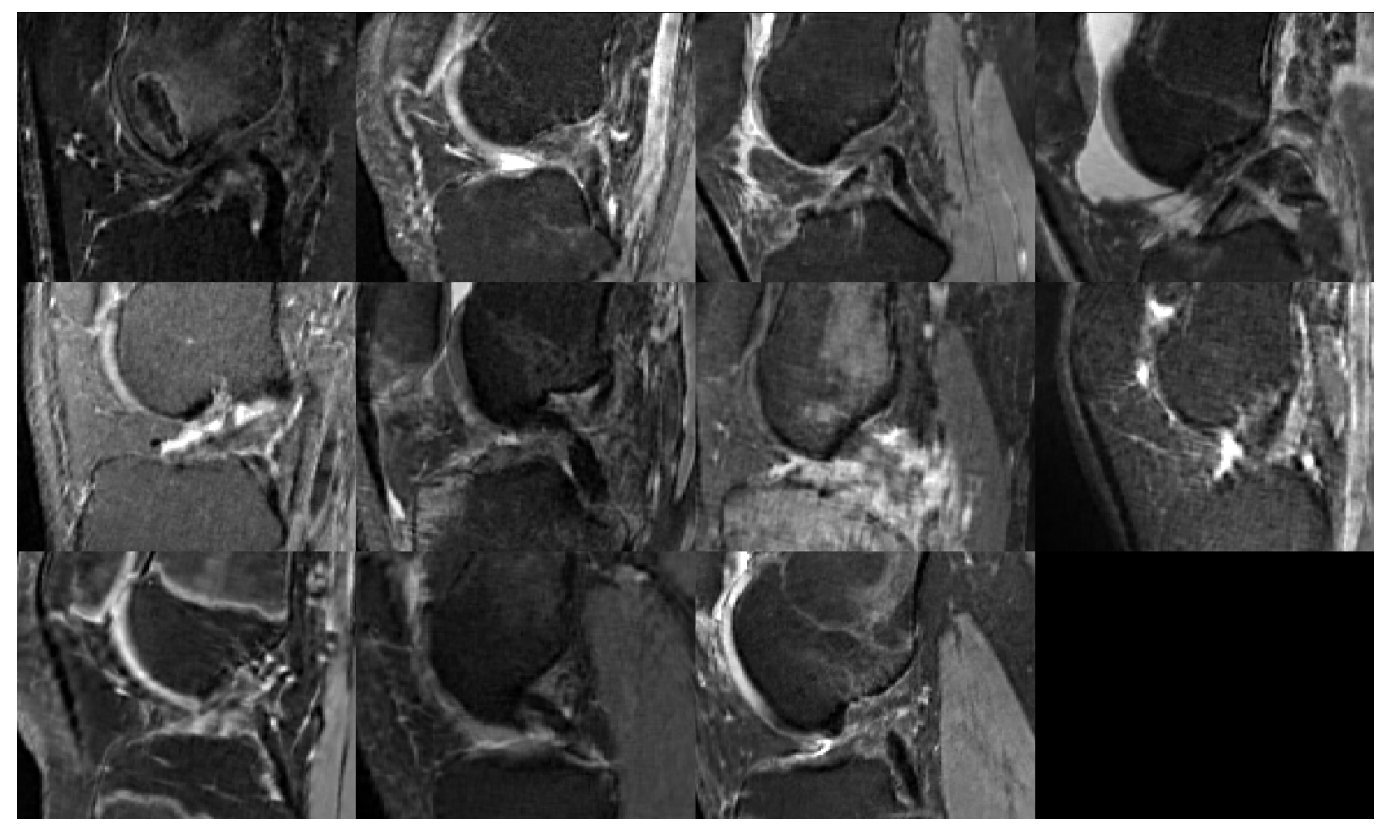

(a) Before standardization.

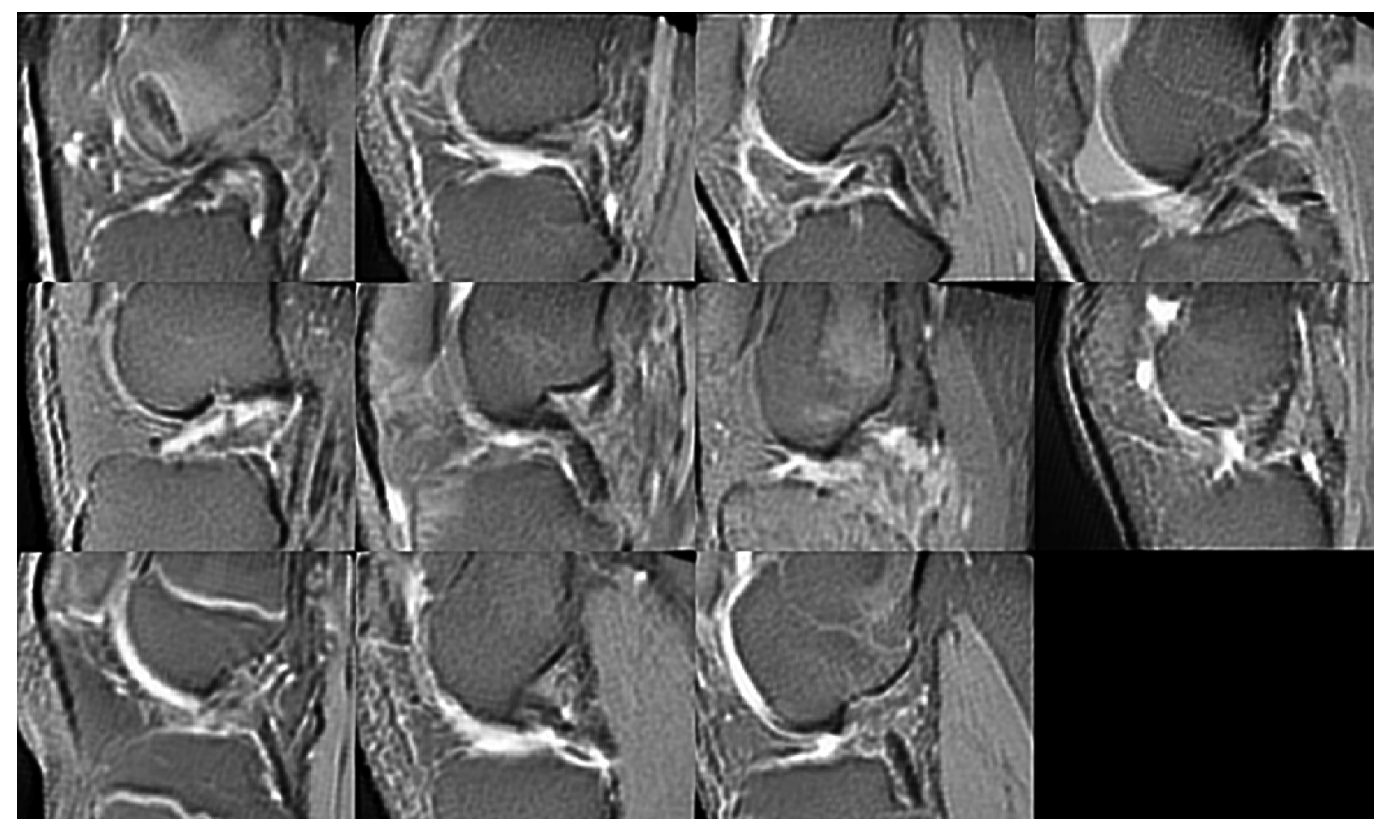

(b) After standardization.

Figure 1: Effect of GAN-based image standardization on a heterogeneous dataset of 11 knee T1-weighted MR images. Image $\mathcal{I}_{05}$ (left column, middle row) is chosen for target. 


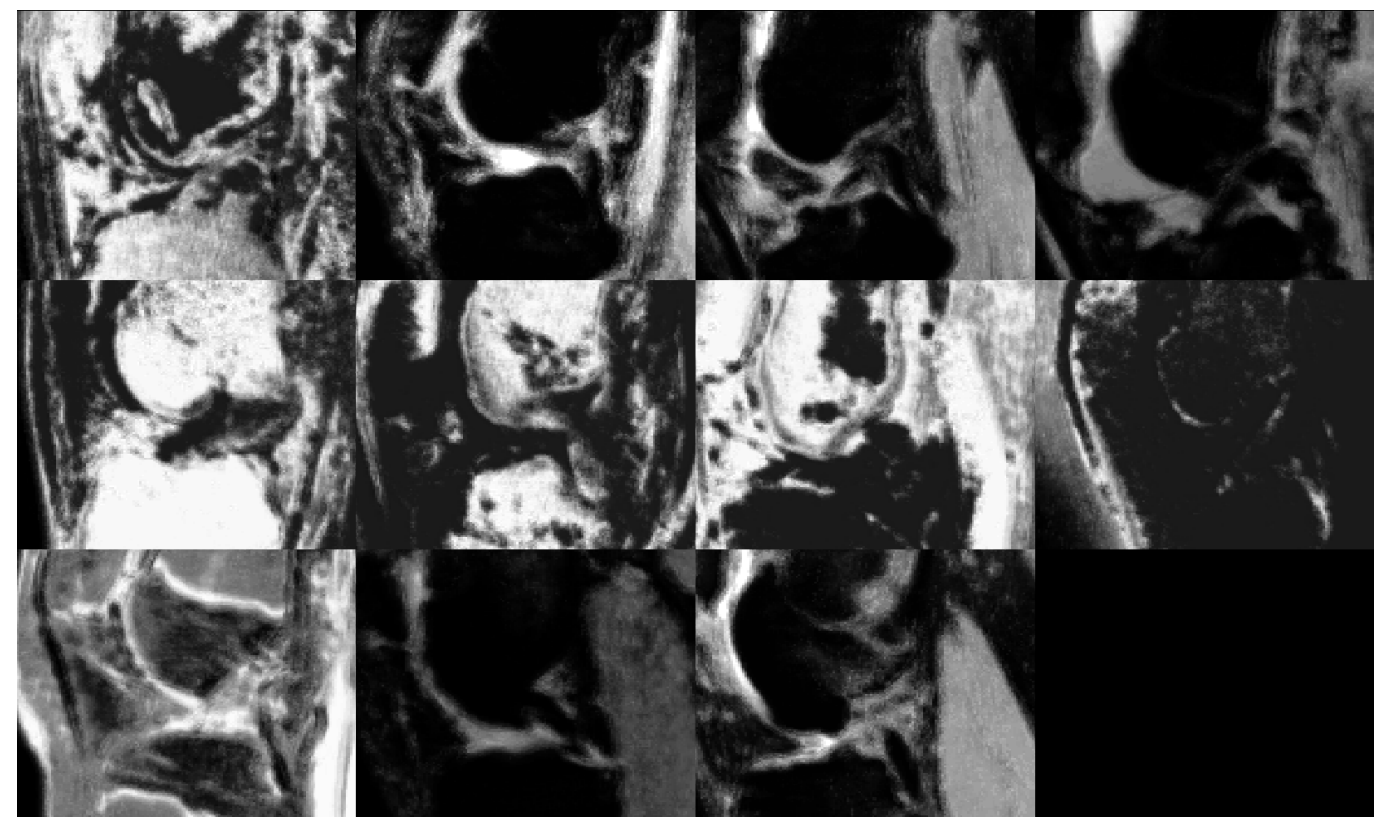

(a) Bone tissue probability before proposed image standardization approach.

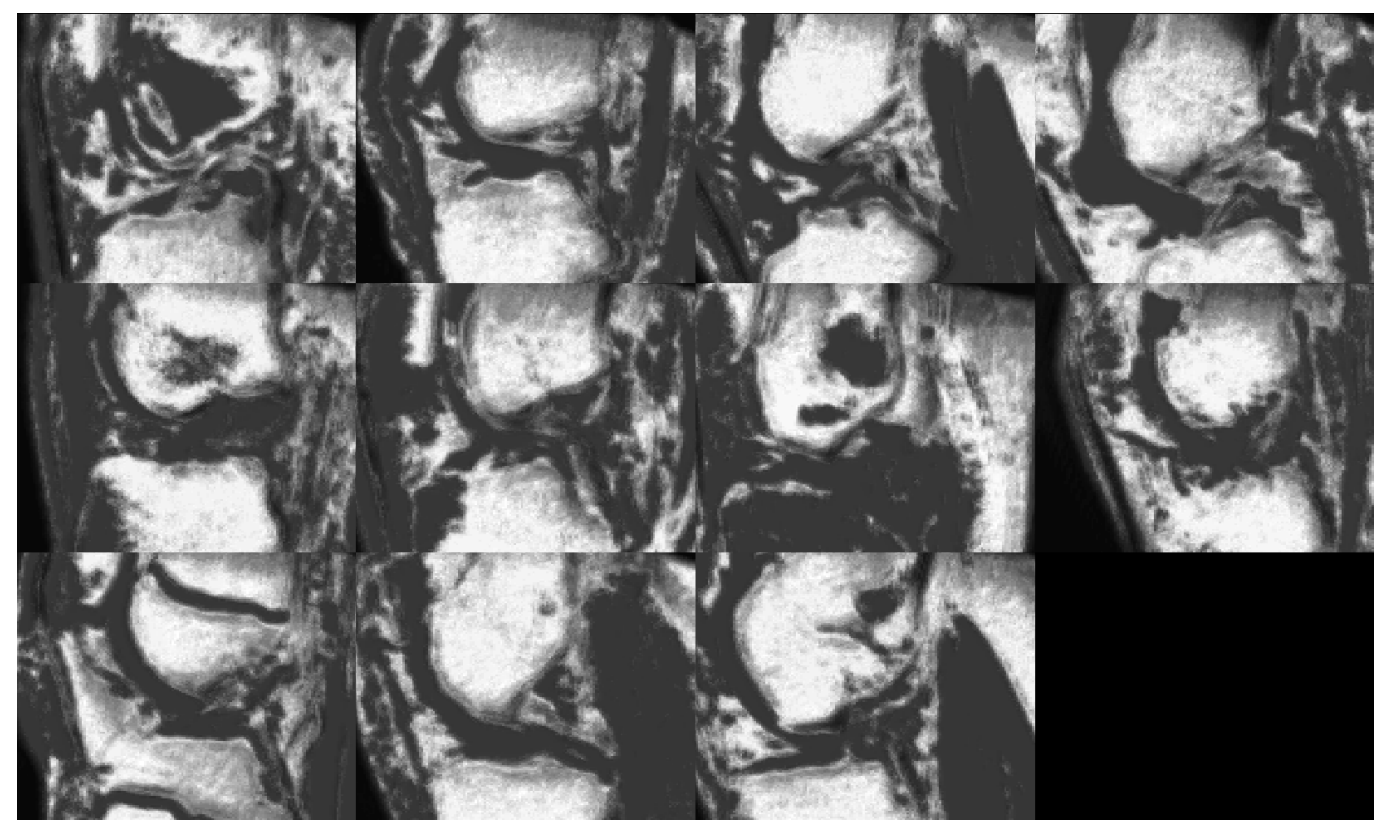

(b) Bone tissue probability after proposed image standardization approach.

Figure 2: Corresponding bone tissue probability maps using a random forest classifier trained on before and after image standardization. 
[2] Jean-Philippe Fortin, Elizabeth M Sweeney, John Muschelli, Ciprian M Crainiceanu, Russell T Shinohara, Alzheimer's Disease Neuroimaging Initiative, et al. Removing inter-subject technical variability in magnetic resonance imaging studies. NeuroImage, 132:198-212, 2016.

[3] Alejandro F Frangi, Sotirios A Tsaftaris, and Jerry L Prince. Simulation and synthesis in medical imaging. IEEE transactions on medical imaging, 37(3):673-679, 2018.

[4] Clément Hognon, Florent Tixier, Olivier Gallinato, Thierry Colin, Dimitris Visvikis, and Vincent Jaouen. Standardization of multicentric image datasets with generative adversarial networks. In IEEE Nuclear Science Symposium and Medical Imaging Conference 2019, 2019.

[5] Tero Karras, Timo Aila, Samuli Laine, and Jaakko Lehtinen. Progressive growing of gans for improved quality, stability, and variation. arXiv preprint arXiv:1710.10196, 2017.

[6] Paul A Yushkevich, Joseph Piven, Heather Cody Hazlett, Rachel Gimpel Smith, Sean Ho, James C Gee, and Guido Gerig. User-guided 3d active contour segmentation of anatomical structures: significantly improved efficiency and reliability. Neuroimage, 31(3):1116-1128, 2006.

[7] Jun-Yan Zhu, Taesung Park, Phillip Isola, and Alexei A Efros. Unpaired image-to-image translation using cycle-consistent adversarial networks. In Proceedings of the IEEE international conference on computer vision, pages 2223-2232, 2017. 